\title{
Proposal for All-Optical Switchable and Tunable Ultrawideband Monocycle Generation Utilizing SOA Wavelength Conversion and Time Delay
}

\author{
Zhefeng HU*, Jianhui XU, and Min HOU \\ Key Laboratory of Optical Fiber Sensing \& Communications (Ministry of Education), School of Communication and \\ Information Engineering, University of Electronic Science and Technology of China, Chengdu, 611731, China \\ *Corresponding author: Zhefeng HU E-mail: zfhu@uestc.edu.cn
}

\begin{abstract}
An all-optical ultrawideband monocycle generator based on wavelength conversion in a semiconductor optical amplifier (SOA) and optical tunable delay in an optical delay line (ODL) is proposed and simulated. The system achieves optically switchable in pulse polarity and tunable in both the pulsewidth and radio frequency (RF) spectrum.
\end{abstract}

Keywords: Microwave photonics; ultrawideband (UWB); tunable; switchable; semiconductor optical amplifier (SOA); tunable time delay

Citation: Zhefeng HU, Jianhui XU, and Min HOU, "Proposal for All-Optical Switchable and Tunable Ultrawideband Monocycle Generation Utilizing SOA Wavelength Conversion and Time Delay," Photonic Sensors, 2017, 7(1): 66-71.

\section{Introduction}

As a promising technology, ultrawideband (UWB) is regarded to have wide applications for short-range, high-capacity wireless communication systems and broadband sensor networks [1]. As defined by the U.S. Federal Communications Commission (FCC), UWB signals must occupy a 10-dB bandwidth more than $500 \mathrm{MHz}$, or a fractional bandwidth greater than $20 \%$ within the spectra from $3.1 \mathrm{GHz}$ to $10.6 \mathrm{GHz}$ [2]. UWB signal generators, which achieve pulse polarity switchability and pulsewidth or radio frequency (RF) tunability, are especially desired to provide wide applications and the flexibility of the systems.

However, the UWB signal suffers tremendous loss in free-space transmission, so it is typically used in short-distance communications. UWB signal generation in the optical domain is recently

Received: 10 November 2016 / Revised: 3 December 2016

(C) The Author(s) 2016. This article is published with open access at Springerlink.com

DOI: 10.1007/s13320-016-0389-6

Article type: Regular developed rapidly, because of the low loss over long-distance transmission in the optical fiber. Many electro-optical methods [3-6] and all-optical methods [7-9] have been proposed. Particularly, an optically switchable and tunable scheme has been demonstrated using the semiconductor optical amplifier (SOA) and single-mode fiber (SMF), but a long span of optical fiber should be used [10].

In the present paper, a novel scheme for all-optical UWB monocycle generator based on wavelength conversion in the SOA and time delay in the optical delay line (ODL) is proposed and simulated. The proposed scheme is optically switchable in pulse polarity and tunable in both the pulsewidth and RF spectrum without a long span of optical fiber.

\section{Operation principle}

The working principle of the optically 
switchable and tunable UWB generation scheme is shown in Fig. 1. A Gaussian pulse train at the wavelength $\lambda_{s}$ is used as the pump. A continuous wave $(\mathrm{CW})$ probe at $\lambda_{p}$ is injected into an SOA with the pump signal together through a wavelength division multiplexer (WDM). Through the effect of cross-gain modulation (XGM), a polarity-reversed Gaussian pulse train is modulated onto the $\mathrm{CW}$ probe. After that, the Gaussian pulse train and polarity-reversed Gaussian pulse train are divided to different beam paths by another WDM. A time delay relative to the output pump is introduced to the output probe by an ODL. By using the third WDM, the output pump and probe are coupled again. Thus, the mixed signal obtains a UWB monocycle shape.

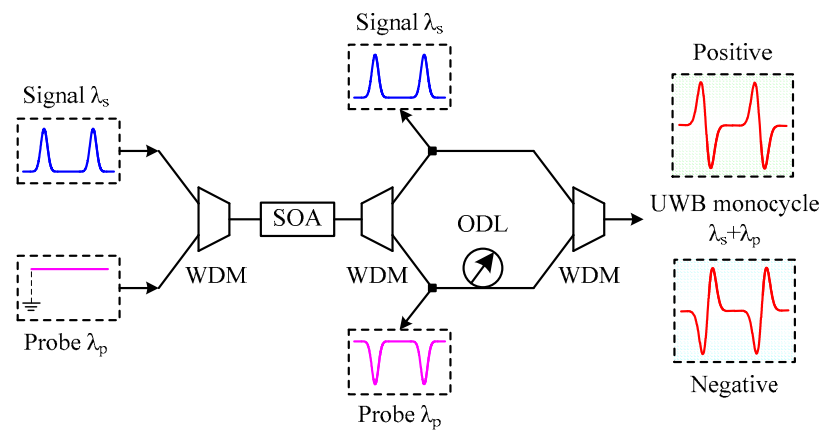

Fig. 1 Schematic illustration for the switchable and tunable UWB monocycle generator.

The time delay between the Gaussian and polarity-reversed Gaussian pulse trains can be controlled by tuning the ODL. Then the pulsewidth of the output UWB monocycle signal will vary. When we tune the ODL to achieve time delay and advance, the generated pulses can be switched between the positive and negative monocycles.

\section{Theoretical model and simulation parameters}

The XGM process in the SOA can be described by the carrier rate equation and propagation equation [11]:

$$
\left\{\begin{array}{c}
\frac{\partial N}{\partial t}=\frac{I}{e V}-R(N)-\sum_{\omega=s, p} \frac{\Gamma g_{\omega}(N)}{A_{0} h c / \lambda_{\omega}}\left|A_{\omega}\right|^{2} \\
\frac{d A_{\omega}}{d z}=\left[\Gamma g_{\omega}(N)(1-\mathrm{i} \alpha)-\alpha_{\mathrm{int}}\right] A_{\omega}
\end{array}\right.
$$

where $N$ is the carrier density, $I$ is the injection current, $e$ is the quantity of electron charge, $V$ and $A_{0}$ are the volume and cross section of the active region, $g_{\omega}(N)$ and $h c / \lambda_{\omega}$ are the gain coefficient and the photon energy at the wavelength $\lambda_{\omega}$, respectively, and the index $\omega$ corresponds to different input lights, including the pump and the probe. $R(N)$ accounts for the carrier consumption due to the radiative recombination and nonradiative recombination. $A_{\omega}$ is the amplitudes at the wavelength $\lambda_{\omega}$.

The recombination rate depends on the carrier density and is given by

$$
R(N)=A N+B N^{2}+C N^{3}
$$

The gain coefficient can be expressed as

$$
\begin{aligned}
g_{\omega}\left(N, \lambda_{\omega}\right)= & a_{1}\left(N-N_{0}\right)-a_{2}\left(\lambda_{\omega}-\lambda_{N}\right)^{2} \\
& +a_{3}\left(\lambda_{\omega}-\lambda_{N}\right)^{3}
\end{aligned}
$$

where $\lambda_{N}$ is the peak wavelength, which is assumed to shift linearly with the carrier density, i.e., $\lambda_{N}=\lambda_{0}-a_{4}\left(N-N_{0}\right)$.

The description and value of SOA parameters used in simulation are listed in Table 1, where $V=l w d$ and $A_{0}=w d$. The pump signal is the Gaussian pulse at $1535 \mathrm{~nm}$ with full width at half maximum (FWHM) of $70 \mathrm{ps}$ and the repetition rate of $1.25 \mathrm{GHz}$. The probe wavelength is $1550 \mathrm{~nm}$. The probe power and pump peak power are $1 \mathrm{~mW}$.

Table 1 SOA parameters used in simulation.

\begin{tabular}{clc}
\hline Symbol & \multicolumn{1}{c}{ Description } & Value \\
\hline$l$ & Length of active region & $10^{-3} \mathrm{~m}$ \\
$w$ & Width of active region & $1.5 \times 10^{-6} \mathrm{~m}$ \\
$d$ & Thickness of active region & $2 \times 10^{-7} \mathrm{~m}$ \\
$A$ & Unimolecular recombination constant & $1.5 \times 10^{8} \mathrm{~s}^{-1}$ \\
$B$ & Bimolecular recombination constant & $1 \times 10^{-16} \mathrm{~m}^{3} / \mathrm{s}$ \\
$C$ & Auger recombination constant & $1.2 \times 10^{-40} \mathrm{~m}^{6} / \mathrm{s}$ \\
$a_{1}$ & Gain constant & $5 \times 10^{-20} \mathrm{~m}^{2}$ \\
$a_{2}$ & Gain constant & $7.4 \times 10^{18} \mathrm{~m}^{-3}$ \\
$a_{3}$ & Gain constant & $3.155 \times 10^{25} \mathrm{~m}^{-4}$ \\
$a_{4}$ & Gain constant & $3 \times 10^{-32} \mathrm{~m}^{4}$ \\
$N_{0}$ & Carrier density at transparency & $1 \times 10^{24} \mathrm{~m}^{-3}$ \\
$\lambda_{0}$ & Wavelength at transparency & $1.56 \times 10^{-6} \mathrm{~m}$ \\
$\alpha$ & Linewidth enhancement factor & 4 \\
$\alpha_{\text {int }}$ & Internal loss & $5 \times 10^{3} \mathrm{~m}^{-1}$ \\
$\Gamma$ & Confinement factor & 0.3 \\
\hline
\end{tabular}




\section{Simulation results and discussion}

Figure 2 shows the waveform of input Gaussian signal, output pump, and output probe, respectively.

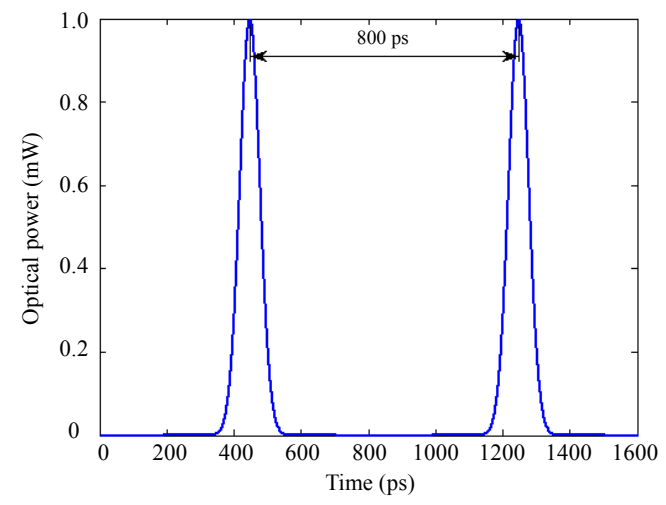

(a)
The FWHMs of the output pump pulse and output probe polarity-reversed pulse are $90.7 \mathrm{ps}$ and $88.1 \mathrm{ps}$, respectively.

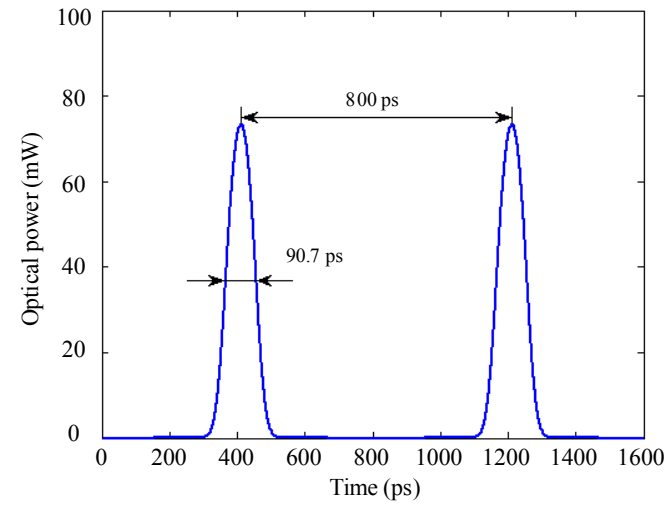

(b)

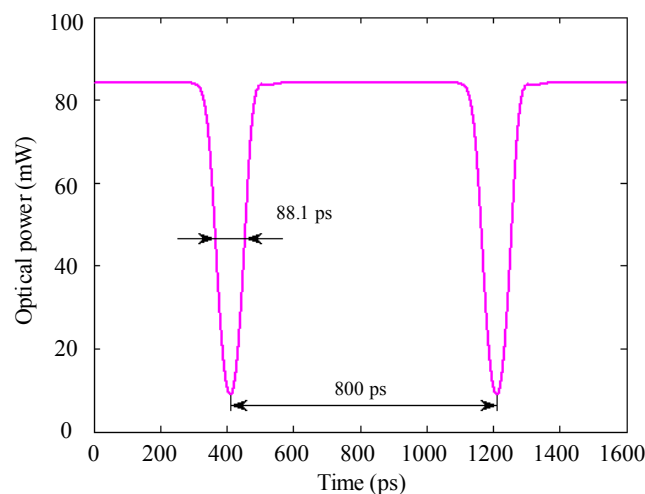

(c)

Fig. 2 Waveforms of different lights: (a) input pump, (b) output pump, and (c) output probe.

Figure 3 depicts the temporal traces of the generated UWB monocycle, when the time delay is tuned at different values. In Figs. 3(a) - 3(c), the time delays are positive. Thus, the output probe drops behind the output pump. The positive UWB monocycle pulses are obtained. However, in Figs. 3(d) - 3(f), the time delays are negative. That is to say, the time advances are achieved. Then the output probe is ahead of the pump. We realize negative UWB monocycle pulses. The repetition period of output UWB signal is $800 \mathrm{ps}$.

The dependence of the generated monocycle pulsewidth on the time delay is illustrated in Fig. 4. The negative time delays represent time advances.
For the generated positive UWB monocycle signal, the upper FWHM and lower FWHM can be tuned from $49.5 \mathrm{ps}$ to $90.1 \mathrm{ps}$ and from $50.4 \mathrm{ps}$ to $87.5 \mathrm{ps}$, respectively. The corresponding parameters of the generated negative UWB monocycle signal can be tuned from $51.8 \mathrm{ps}$ to $90.6 \mathrm{ps}$ and from $55.8 \mathrm{ps}$ to $87.5 \mathrm{ps}$, respectively.

To further confirm the successful realization of the UWB monocycle generation, Fig. 5 denotes the radio frequency $(\mathrm{RF})$ spectra and their envelopes for the generated UWB monocycle signals. From the line space, we find the repetition frequency of the output UWB signal is $1.25 \mathrm{GHz}$. 


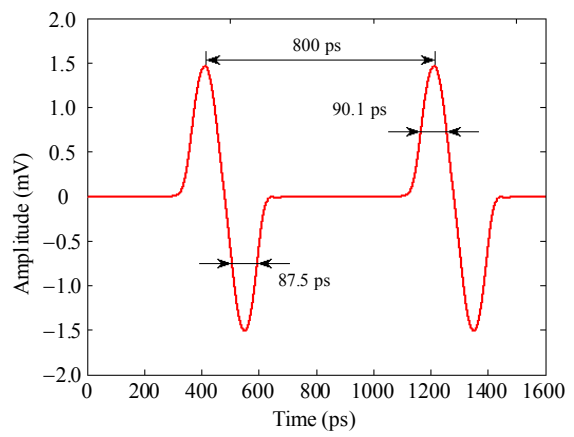

(a)

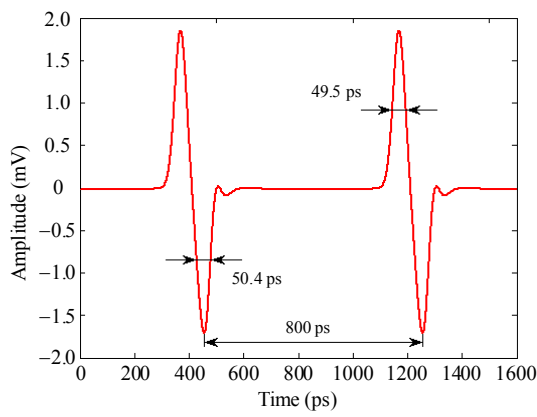

(c)

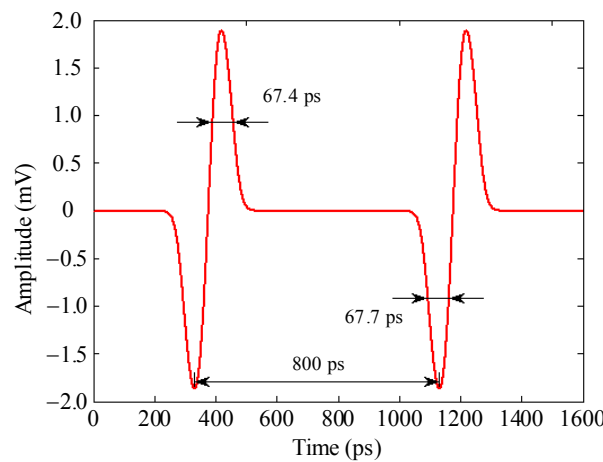

(e)

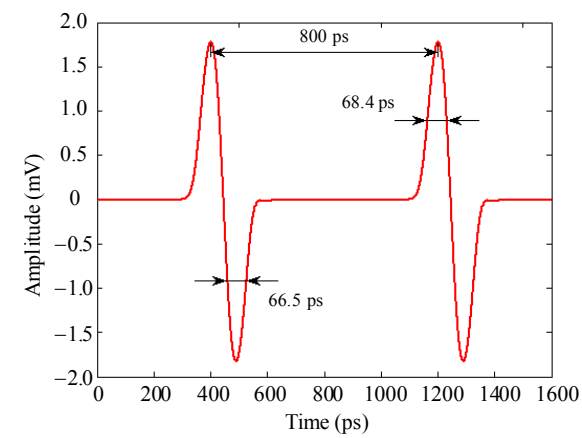

(b)

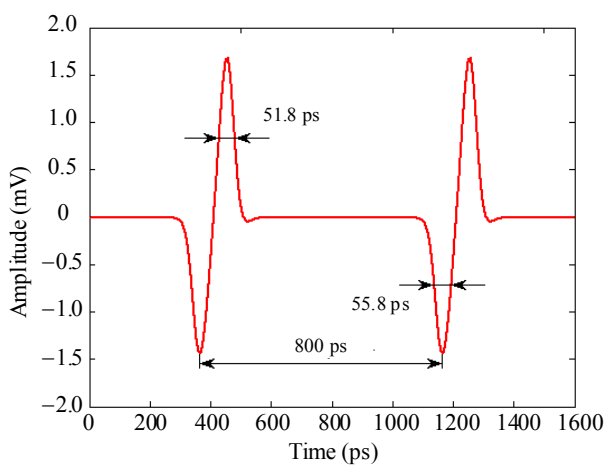

(d)

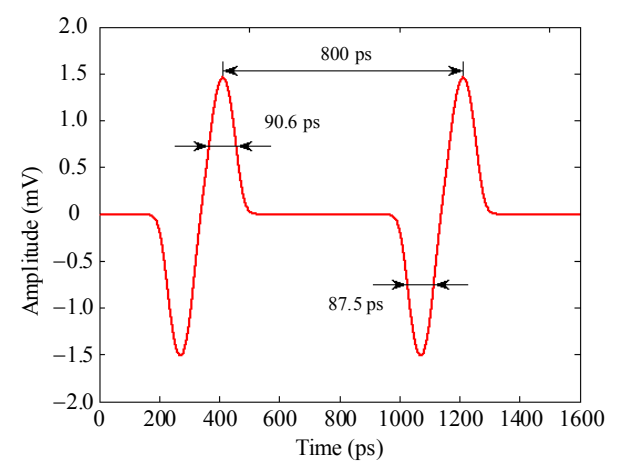

(f)

Fig. 3 Temporal traces of the generated UWB monocycle signals with different time delays [(a) - (c): $140 \mathrm{ps,} 70 \mathrm{ps,}$ and $10 \mathrm{ps}]$ and advances [(d) - (f): $-10 \mathrm{ps},-70 \mathrm{ps}$, and $-140 \mathrm{ps}]$ : (a) - (c) positive UWB monocycle and (d) - (f) negative UWB monocycle.

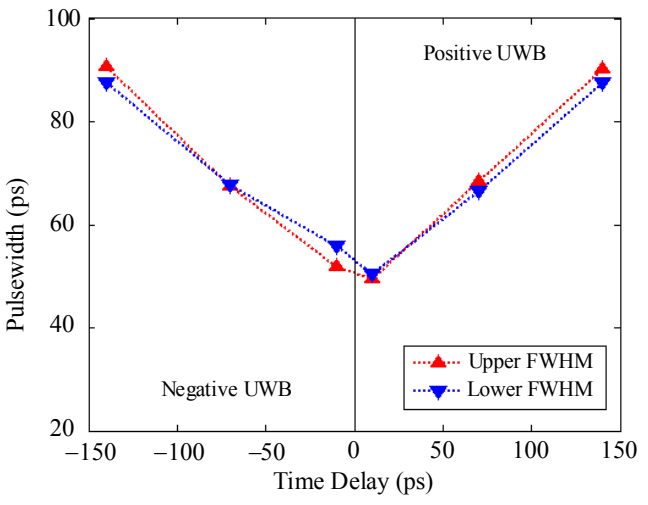

Fig. 4 Pulsewidth depending on the time delay.

Figure 6 plots the variation in the RF spectrum when the time delay or advance is varying. From Fig. 6(a), we know that the central frequency and 10-dB bandwidth of the generated positive UWB monocycle can be tuned from $3.30 \mathrm{GHz}$ to $5.40 \mathrm{GHz}$ and from $5.34 \mathrm{GHz}$ to $8.86 \mathrm{GHz}$, respectively; for the negative UWB monocycle, they can be tuned from $3.24 \mathrm{GHz}$ to $5.11 \mathrm{GHz}$ and from $5.23 \mathrm{GHz}$ to $8.30 \mathrm{GHz}$, respectively. Figure 6(b) shows that, the fractional bandwidth of the generated monocycle signal have very slight fluctuation between $161.4 \%$ 
and $164.1 \%$. It is noted that the obtained RF spectra

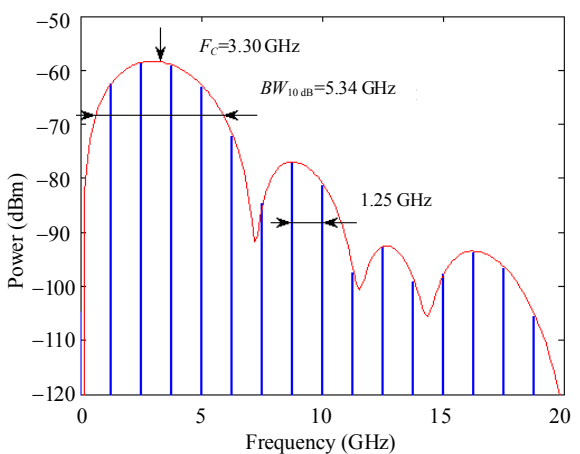

(a)

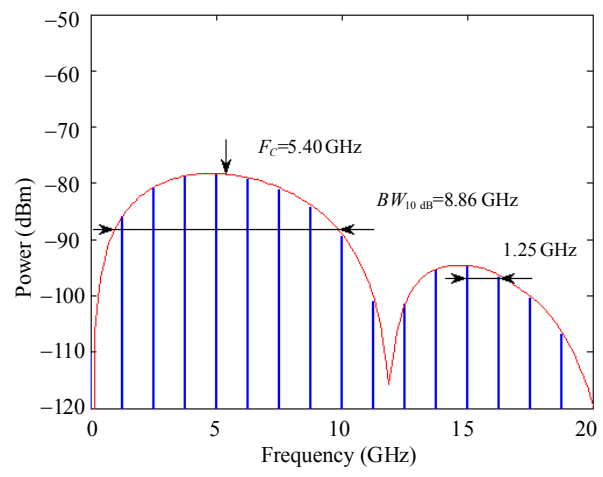

(c)

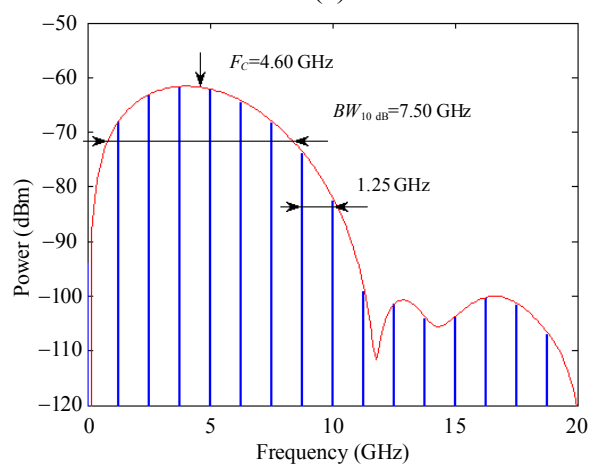

(e) are in good agreement with the FCC UWB definition.

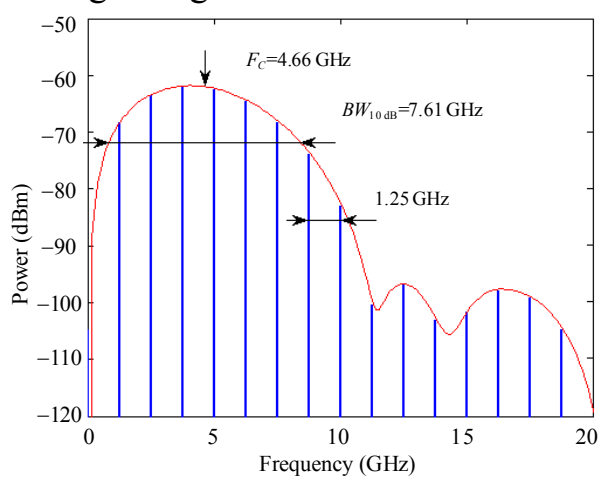

(b)

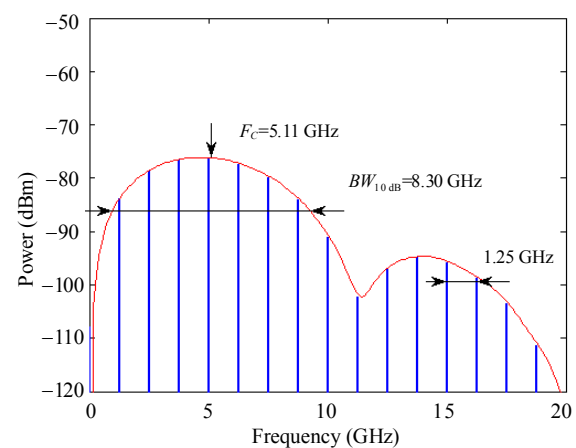

(d)

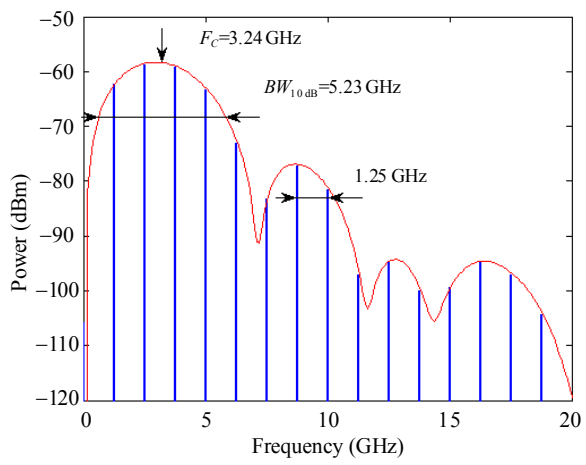

(f)

Fig. 5 RF spectra for UWB monocycle pulses with different time delays [(a) - (c): $140 \mathrm{ps}, 70 \mathrm{ps}$, and $10 \mathrm{ps}]$ and advances [(d) - (f): $-10 \mathrm{ps},-70 \mathrm{ps}$, and $-140 \mathrm{ps}$ ]: (a) - (c) positive UWB monocycle and (d) - (f) negative UWB monocycle.

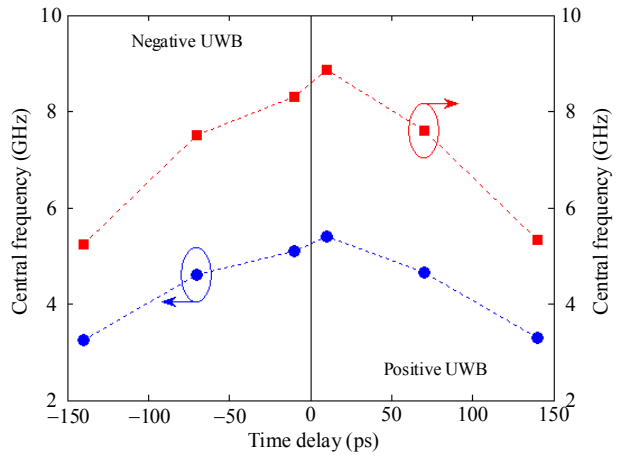

(a)

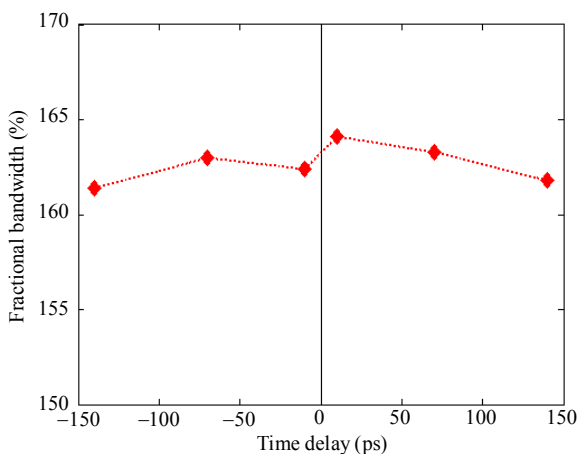

(b)

Fig. 6 Tunability in RF spectrum: (a) central frequency, 10-dB bandwidth and (b) fractional bandwidth as a function of the time delay.

By synthesizing the results shown in Figs. 3, 4, 5, and 6, we find that when the time delay increases, 
the upper and lower FWHMs of the generated positive UWB monocycle signal will increase, but the central frequency and $10-\mathrm{dB}$ bandwidth will decrease. When the time advance increases, the upper and lower FWHMs of the generated negative UWB monocycle will increase, but the central frequency and 10-dB bandwidth will decrease. Thus, we realize pulse polarity switchability, and both pulsewidth and RF spectrum tunability for the generated UWB monocycle signal, by controlling the time delay and advance.

For the UWB signal, the pulse polarity modulation (PPM) is regarded as an important modulation scheme [12]. The proposed optically switchable monocycle generator can be expediently used for PPM for the optical UWB signal. And the optical tunability of the proposed monocycle generator offers the convenience to control the pulsewidth and the RF band for a flexible system.

\section{Conclusions}

We have proposed and numerically demonstrated an all-optical UWB monocycle generator. Our scheme is optically switchable in pulse polarity and tunable in both the pulsewidth and RF spectrum. The pulsewidth, central frequency, and $10-\mathrm{dB}$ bandwidth of the generated monocycle signal can vary expediently by tuning the ODL. The proposed monocycle generator provides a potential method for PPM for the optical UWB signal and also provides flexibility for the applications of the UWB technology.

\section{Acknowledgment}

This work was supported by the National Natural Science Foundation of China under grant 61501088, 61307088, and 61675040, and the Fundamental Research Funds for the Central Universities under grant ZYGX2014J006.

Open Access This article is distributed under the terms of the Creative Commons Attribution 4.0 International License (http://creativecommons.org/ licenses/by/4.0/), which permits unrestricted use, distribution, and reproduction in any medium, provided you give appropriate credit to the original author(s) and the source, provide a link to the Creative Commons license, and indicate if changes were made.

\section{References}

[1] D. Porcine, P. Research, and W. Hirt, "Ultra-wideband radio technology: potential and challenges ahead," IEEE Communications Magazine, 2003, 41(7): 66-74.

[2] G. R. Aiello and G. D. Rogerson, "Ultra-wideband wireless systems," IEEE Communications Magazine, 2003, 4(2): 36-47.

[3] Q. Wang and J. Yao, "UWB doublet generation using nonlinearly-biased electro-optic intensity modulator," Electronics Letters, 2006, 42(22): 1304-1305.

[4] F. Zeng and J. Yao, "Ultrawideband impulse radio signal generation using a high-speed electrooptic phase modulator and a fiber-Bragg-grating-based frequency discriminator," IEEE Photonics Technology Letters, 2006, 18(19), 2062-2064.

[5] Q. Wang and J. Yao, "Switchable optical UWB monocycle and doublet generation using a reconfigurable photonic microwave delay-line filter," Optics Express, 2007, 15(22): 14667-14672.

[6] Q. Wang and J. Yao, "An electrically switchable optical ultrawideband pulse generator," Journal of Lightwave Technology, 2007, 25(11): 3626-3633.

[7] Q. Wang, F. Zeng, S. Blais, and J. Yao, "Optical ultrawideband monocycle pulse generation based on cross-gain modulation in a semiconductor optical amplifier," Optics Letters, 2006, 31(21): 3083-3085.

[8] J. Dong, X. Zhang, J. Xu, and D. Huang, "Ultrawideband monocycle generation using cross-phase modulation in a semiconductor optical amplifier," Optics Letters, 2007, 32(10): 1223-1225.

[9] J. Dong, X. Zhang, J. Xu, and D. Huang, “All-optical ultrawideband monocycle generation utilizing gain saturation of a dark return-to-zero signal in a semiconductor optical amplifier," Optics Letters, 2007, 32(15): 2158-2160.

[10] J. Hsieh, P. Gong, S. Lee, and J. Wu, "Improved dynamic characteristics on four-wave mixing wavelength conversion in light-holding SOAs," IEEE Journal of Selected Topics on Quantum Electronics, 2004, 10(5): 1187-1196.

[11] Z. Hu, J. Sun, J. Shao, and X. Zhang, "Filter-free optically switchable and tunable ultrawideband monocycle generation based on wavelength conversion and fiber dispersion," IEEE Photonics Technology Letters, 2010, 22(1): 42-44.

[12] J. Yao, F. Zeng, and Q. Wang, "Photonic generation of ultrawideband signals," Journal of Lightwave Technology, 2007, 25(11): 3219-3235. 